\title{
Genes expression related to the effects of hypoxia in the marine mussel, Mytilus galloprovincialis
}

\author{
Ekaterina Vodiasova \\ IBSS RAS, Sevastopol, Russia \\ eavodiasova@gmail.com \\ Yakov Meger \\ SSU, Sevastopol, Russia \\ meger_yakov@mail.ru
}

\author{
Aleksandra Andreyeva \\ IBSS RAS, Sevastopol, Russia \\ andreevaal@gmail.com \\ Irina Deghtyar \\ SSU, Sevastopol, Russia \\ skuratovskaya95@mail.ru
}

\author{
Anastasiya Lantushenko \\ SSU, Sevastopol, Russia \\ lantushenko@mail.ru \\ Dmitry Afonnikov \\ ICG SB RAS, Novosibirsk, Russia \\ ada@bionet.nsc.ru
}

\begin{abstract}
Despite that mussel is an important object of mariculture and can play the role of an ecosystem bio-indicator, the study of the effect of hypoxia on these molluses is limited only by biochemical and RT-PCR experiments for individual genes (enzymes). There is no data on changes in the expression profile of all genes based on RNA sequencing. At the same time, an understanding of physiological processes requires comprehensive studies based on the study of the expression of all genes in target tissues. At this work, the transcriptomes of the gills of Mytilus galloprovincialis exposed to hypoxic stress (DO $0.3 \mathrm{mg} / \mathrm{l}$ ) for $24 \mathrm{~h}$ were sequenced using Illumina technology for the first time. The raw reads assembled into 255,302 transcripts with an $\mathrm{N} 50$ value of $1,273 \mathrm{nt}$. A total of 65 transcripts that differed in abundance in the hypoxia-exposed mussels were identified. Their putative function was assigned using BLAST. The data obtained will allow further large-scale studies for other targeted tissues and to study all aspects of the physiological response of $M$. galloprovincialis to the emerging oxygen deficiency in the environment.
\end{abstract}

Keywords - short-time hypoxia, Mytilus galloprovincialis, transcriptomic, differential expression, RNA-seq, comparative analyses

\section{Motivation and aim}

There is a lack of works based on transcriptomics data about the expression of genes in mussels hypoxia-exposed. Most of them are devoted to the study of changes in the activity of the enzymes and their concentration in various tissues [1-4]. Very few studies analyzing gene expression changes using RT-PCR methods due to the insufficient nucleotide sequences of the target genes [2, 5, 6], transcriptomics data on the influence of hypoxia is absent. Thus, the analysis of differentially expressed genes will probably allow to identify new physiological processes that are activated in the cells of target tissues at the molecular level.

\section{Methods}

Mussels, M. galloprovincialis (shell length 56.3+/- 1.5 $\mathrm{mm}$, weight $12.7+/-2.1 \mathrm{~g}, \mathrm{n}=16$ ) were placed in a tank with seawater. Hypoxic conditions were conducted by bubbling of the water with nitrogen gas reaching the concentration 0.3 $\mathrm{mg} / \mathrm{l}$. Oxygen concentration was controlled on oxygen sensor (Ohaus, USA). After $24 \mathrm{~h}$ incubation in hypoxia, gill tissue was obtained and RNA was extracted using Zymo Research Quick RNA mini extraction kit (R1050, Zymo Research). Total RNA was isolated from gill tissues of mussel from control (4 ind.) and experimental (3 ind.) group. The libraries were synthesized with TruSeq Stranded Total RNA Library Prep (20020596, Illumina) and single-end sequencing as 150 nt reads was done on Illumina NextSeq 500 instrument.
Quality and length trimming of the reads was conducted using Fastp v. 0.20.0. The cleaned sequencing reads were then de novo assembled using Trinity v. 2.0.6 with default parameters. Sequence similarity and annotation of transcripts was performed using local BLAST v. 2.9.0+. The predicted protein regions were mapped to Gene Ontology (GO) and Pfam. GO was used to get an overview of the proportion of enriched GO terms between normoxia and hypoxia.

\section{Results}

Differentially expressed genes in gills during oxygen depletion stress were obtained based on total RNA-seq. The raw reads assembled into 255,302 transcripts with an N50 value of 1,273 nt. Changes in gene expression in control compared to experimental groups were estimated. A total of 65 transcripts that differed in abundance in the hypoxiaexposed mussels were identified. The GO analysis was performed on the differentially expressed transcripts of mussels inside the control and experimental group. Besides, a qualitative comparative analysis was carried out on 50 differentially expressed genes that were identified in gill tissues for M. galloprovincialis exposed to hypoxic stress (DO $2 \mathrm{mg} / \mathrm{l}$ ) for $24 \mathrm{~h}$.

The obtained data will allow to further study in more detail all aspects of the physiological response of $M$. galloprovincialis to the emerging oxygen deficiency in the environment.

\section{ACKNOWLEDGMENT}

This work is funded by State Assignment (state registration number N 0828-2018-0003), the Ministry of education and Science of the Russian Federation grant No. 14.W03.31.0015, and by RSF grant 18-14-00086 and an internal grant of SSU for 2020 No. 33/06-31.

\section{REFERENCES}

[1] Anestis, A., Pörtner, H. O., \& Michaelidis, B. (2010). Anaerobic metabolic patterns related to stress responses in hypoxia exposed mussels Mytilus galloprovincialis. Journal of experimental marine biology and ecology, 394(1-2), 123-133.

[2] Woo, S., Denis, V., Won, H., Shin, K., Lee, G., Lee, T. K., \& Yum, S. (2013). Expressions of oxidative stress-related genes and antioxidant enzyme activities in Mytilus galloprovincialis (Bivalvia, Mollusca) exposed to hypoxia. Zoological Studies, 52(1), 15.

[3] Franco-Martínez, L., Tvarijonaviciute, A., Mateo, S. V., Cerón, J. J., Romero, D., Oliveira, M.,... \& Martínez-Subiela, S. (2019). Evaluation of C-reactive-like protein in Mytilus galloprovincialis. Ecological Indicators, 106, 105537.

[4] Haider, F., Falfushynska, H. I., Timm, S., \& Sokolova, I. M. (2020). Effects of hypoxia and reoxygenation on intermediary metabolite homeostasis of marine intertidal bivalves Mytilus edulis and 
Crassostrea gigas. Comparative Biochemistry and Physiology Part A: Molecular \& Integrative Physiology, 110657.

[5] Woo, S., Jeon, H. Y., Kim, S. R., \& Yum, S. (2011). Differentially displayed genes with oxygen depletion stress and transcriptional responses in the marine mussel, Mytilus galloprovincialis. Comparative Biochemistry and Physiology Part D: Genomics and Proteomics, 6(4), 348-356.
[6] Giannetto, A., Maisano, M., Cappello, T., Oliva, S., Parrino, V., Natalotto, A.,... \& Fasulo, S. (2015). Hypoxia-inducible factor $\alpha$ and Hif-prolyl hydroxylase characterization and gene expression in shorttime air-exposed Mytilus galloprovincialis. Marine biotechnology, 17(6), 768-781. 